\title{
ON EXPONENTIAL FUNCTIONALS OF PROCESSES WITH INDEPENDENT INCREMENTS
}

\author{
P. Salminen, Department of Natural Sciences Åbo \\ Akademi University FIN-20500 Åbo Finland \\ and \\ L. Vostrikova ${ }^{1}$, LAREMA, Département de \\ Mathématiques, Université d'Angers, 2, Bd Lavoisier \\ 49045, Angers Cedex 01
}

\begin{abstract}
In this paper we study the exponential functionals of the processes $X$ with independent increments, namely

$$
I_{t}=\int_{0}^{t} \exp \left(-X_{s}\right) d s, \quad t \geq 0
$$

and also

$$
I_{\infty}=\int_{0}^{\infty} \exp \left(-X_{s}\right) d s
$$

When $X$ is a semi-martingale with absolutely continuous characteristics, we derive recurrent integral equations for Mellin transform $\mathbf{E}\left(I_{t}^{\alpha}\right), \alpha \in \mathbb{R}$, of the integral functional $I_{t}$. Then we apply these recurrent formulas to calculate the moments. We present also the corresponding results for the exponential functionals of Levy processes, which hold under less restrictive conditions then in [7. In particular, we obtain an explicit formula for the moments of $I_{t}$ and $I_{\infty}$, and we precise the exact number of finite moments of $I_{\infty}$.
\end{abstract}

MSC 2010 subject classifications: 60G51, 91G80

\section{IntroduCtion}

The exponential functionals arise in many areas : in the theory of selfsimilar Markov processes, in the theory of random processes in random environments, in the mathematical statistics, in the mathematical finance, in the insurance. In fact, self-similar Markov processes are related with exponential functionals via Lamperti transform, namely

\footnotetext{
${ }^{11}$ This work is supported in part by DEFIMATHS project of the Reseach Federation of "Mathématiques de Pays de la Loire" and PANORisk project of Pays de la Loire region.
} 
self-similar Markov process can be written as an exponential of Levy process time changed by the inverse of exponential functional of the same Levy process (see [21]). In the mathematical statistics the exponential functionals appear, for exemple, in the study of Pitman estimators (see [23]). In the mathematical finance the question is related to the perpetuities containing the liabilities, the perpetuities subjected to the influence of economical factors (see, for example, [18]), and also with the prices of Asian options and related questions (see, for instance, [16] and references therein). In the insurance, this connection is made via the ruin problem, the problem in which the exponential functionals appear very naturally (see, for exemple [25], [1], [17] and references therein).

In the case of Levy processes, the asymptotic behaviour of exponential functionals was studied in [9], in particular for $\alpha$-stable Levy processes. The authors also give an integro-differential equation for the density of the law of exponential functionals, when this density w.r.t the Lebesgue measure exists. In [7], for Levy subordinators, the authors give the formulas for the positive and negative moments, the Mellin transform and the Laplace transform. The questions related with the characterisation of the law of exponential functionals by the moments was also studied. General information about Levy processes can be find in [29], 5], [20].

In more general setting, related to the Lévy case, the following functional

$$
\int_{0}^{\infty} \exp \left(-X_{s-}\right) d \eta_{s}
$$

was studied by many authors, where $X=\left(X_{t}\right)_{t \geq 0}$ and $\eta=\left(\eta_{t}\right)_{t \geq 0}$ are independent Lévy processes. The interest to this functional can be explained by a very close relation between the distribution of this functional and the stationary density of the corresponding generalized Ornshtein-Uhlenbeck process. We recall that the generalized OrnshteinUhlenbeck process $Y=\left(Y_{t}\right)_{t>0}$ verify the following differential equation

$$
d Y_{t}=Y_{t-} d X_{t}+d \eta_{t}
$$

When the jumps of the process $\eta$ are strictly bigger than -1, and $X_{0}=0$, the solution of this equation is

$$
Y_{t}=\mathcal{E}(X)_{t}\left(Y_{0}+\int_{0}^{t} \frac{d \eta_{s}}{\mathcal{E}(X)_{s-}}\right)
$$


where $\mathcal{E}(X)$ is Doléan-Dade exponential (see [15] for the details). Finally, if we introduce another Lévy process $\hat{X}$ such that for all $t>0$

$$
\mathcal{E}(X)_{t}=\exp \left(\hat{X}_{t}\right)
$$

then the integral of the type (11) appear in (33).

The conditions for finiteness of the integral (1) was obtained in [13]. The continuity properties of the law of this integral was studied in [6], where the authors give the condition for absence of the atoms and also the conditions for absolute continuity of the laws of integral functionals w.r.t. the Lebesgue measure. Under the assumptions which ensure the existence of the density of these functionals, the equations for the density are given in [3], 4], [19]. It should be noticed that taking the process $\eta$ being only drift, one can get $I_{\infty}$ as a special case of the Lévy framework.

In the papers 24] and 22], again for Levy process, the properties of the exponential functionals $I_{\tau_{q}}$ was studied where $\tau_{q}$ is independent exponential random variable of the parameter $q>0$. In the article [22] the authors studied the existence of the density of the law of $I_{\tau_{q}}$, they give an integral equation for the density and the asymptotics of the law of $I_{\infty}$ at zero and at infinity, when $X$ is a positive subordinator. The results given in [24] involve analytic Wiener-Hopf factorisation, Bernstein functions and contain the conditions for regularity, semiexplicite expression and asymptotics for the distribution function of the exponential functional killed at the independent exponential time $\tau_{q}$. Despite numerous studies, the distribution properties of $I_{t}$ and $I_{\infty}$ are known only in a limited number of cases. When $X$ is Brownian motion with drift, the distributions of $I_{t}$ and $I_{\infty}$ was studied in [11] and for a big number of specific processes $X$ and $\eta$, like Brownian motion with drift and compound Poisson process, the distributions of $I_{\infty}$ was given in [14].

Exponential functionals for diffusions was studied in [26]. The authors considered exponential functionals stopped at first hitting time and they derive the Laplace transform of these functionals. To find the laws of such exponential functionals, the authors perform a numerical inversion of the corresponding Laplace transform. The relations between the hitting times and the occupation times for the exponential functionals was considered in [28], where the versions of identities in law such as Dufresne's identity, Ciesielski-Taylor's identity, Biane's identity, LeGall's identity was considered. 
Howerever, the exponential functionals involving non-homogeneous processes with independent increments (PII in short) have not been studied sufficiently up to now. Only a few results can be found in the literature. Some results about the moments of the exponential functional of this type are given in [12]. At the same time PII models for logarithme of the prices are quite natural in the mathematical finance, it is the case of the non-homogeneous Poisson process, the Levy process with deterministic time change, the integrals of Lévy processes with deterministic integrands, the hitting times for diffusions and so on (see for instance [30, [10], 2]).

The aim of this paper is to study the exponential functionals of the processes $X$ with independent increments, namely

$$
I_{t}=\int_{0}^{t} \exp \left(-X_{s}\right) d s, \quad t \geq 0
$$

and also

$$
I_{\infty}=\int_{0}^{\infty} \exp \left(-X_{s}\right) d s
$$

and give such important characteristics of these exponential functionals as the moments and the Laplace transforms and the Mellin transforms.

For that we consider a real valued process $X=\left(X_{t}\right)_{t \geq 0}$ with independent increments and $X_{0}=0$, which is a semi-martingale with respect to its natural filtration. We denote by $(B, C, \nu)$ a semi-martingale triplet of this process, which can be chosen deterministic (see [15], Ch. II, p.106). We suppose that $B=\left(B_{t}\right)_{t \geq 0}, C=\left(C_{t}\right)_{t \geq 0}$ and $\nu$ are absolutely continuous with respect to the Lebesgue measure in $t$, i.e. that $X$ is an Ito process such that

$$
B_{t}=\int_{0}^{t} b_{s} d s, \quad C_{t}=\int_{0}^{t} c_{s} d s, \nu(d t, d x)=d t K_{t}(d x)
$$

with the measurable functions $b=\left(b_{s}\right)_{s \geq 0}, c=\left(c_{s}\right)_{s \geq 0}$, and the kernel $K=\left(K_{t}(A)\right)_{t \geq 0, A \in \mathcal{B}(\mathbb{R} \backslash\{0\})}$. For more information about the semimartingales and the Ito processes see [15].

We assume that the compensator of the measure of the jumps $\nu$ verify the usual relation: for each $t \in \mathbb{R}^{+}$

$$
\int_{0}^{t} \int_{\mathbb{R} \backslash\{0\}}\left(x^{2} \wedge 1\right) K_{s}(d x) d s<\infty .
$$


We recall that the characteristic function of $X_{t}$

$$
\phi_{t}(\lambda)=\mathbf{E} \exp \left(i \lambda X_{t}\right)
$$

is defined by the following expression: for $\lambda \in \mathbb{R}$

$\phi_{t}(\lambda)=\exp \left\{i \lambda B_{t}-\frac{1}{2} \lambda^{2} C_{t}+\int_{0}^{t} \int_{\mathbb{R} \backslash\{0\}}\left(e^{i \lambda x}-1-i \lambda x \mathbf{1}_{\{|x| \leq 1\}}\right) K_{s}(d x) d s\right\}$

which can be easily obtained by the Ito formula for semimartingales. We recall also that $X$ is a semi-martingale if and only if for all $\lambda \in \mathbb{R}$ the characteristic function of $X_{t}$ is of finite variation in $t$ on finite intervals (cf. [15], Ch.2, Th. 4.14, p.106 ). Moreover, the process $X$ always can be written as a sum of a semi-martingale and a deterministic function which is not necessarily of finite variation on finite intervals.

From the formula for the characteristic function we can easily find the Laplace transform of $X_{t}$, if it exists:

$$
\mathbf{E}\left(e^{-\alpha X_{t}}\right)=e^{-\Phi(t, \alpha)}
$$

Putting $\lambda=i \alpha$ in the previous formula, we get that

$$
\Phi(t, \alpha)=\alpha B_{t}-\frac{1}{2} \alpha^{2} C_{t}-\int_{0}^{t} \int_{\mathbb{R} \backslash\{0\}}\left(e^{-\alpha x}-1+\alpha x \mathbf{1}_{\{|x| \leq 1\}}\right) K_{s}(d x) d s .
$$

As known, in the case when $X$ is a Levy process with parameters $\left(b_{0}, c_{0}, K_{0}\right)$, it holds

$$
\mathbf{E}\left(e^{-\alpha X_{t}}\right)=e^{-t \Phi(\alpha)}
$$

with

$$
\Phi(\alpha)=\alpha b_{0}-\frac{1}{2} \alpha^{2} c_{0}-\int_{\mathbb{R} \backslash\{0\}}\left(e^{-\alpha x}-1+\alpha x \mathbf{1}_{\{|x| \leq 1\}}\right) K_{0}(d x) .
$$

In this article we derive a simple sufficient condition for finiteness of $\mathbf{E}\left(I_{t}^{\alpha}\right)$ with fixed $t>0$ (see Proposition 1). The main results of the paper, presented in Theorems 1 and 2, give recurrent integral equations for Mellin transform $\mathbf{E}\left(I_{t}^{\alpha}\right)$. The starting point for the proof of these results is time reversal of the process $X$ at fixed time $t>0$, i.e. we introduce a new process $Y^{(t)}=\left(Y_{s}^{(t)}\right)_{0 \leq s \leq t}$ with $Y_{s}^{(t)}=X_{t}-X_{(t-s)-}$. In Lemma 1 we show that

$$
I_{t}=e^{-Y_{t}^{(t)}} \int_{0}^{t} e^{Y_{s}^{(t)}} d s
$$

Then, we prove that $Y^{(t)}$ is a process with independent increments and we identify its semi-martingale characteristics. This time reversal plays an important role and permits to replace the process $\left(I_{t}\right)_{t \geq 0}$ which is not Markov, by a family of Markov processes $V^{(t)}=\left(V_{s}^{(t)}\right)_{0 \leq s \leq t}$ indexed 
by $t>0$, where $V_{s}^{(t)}=e^{-Y_{s}^{(t)}} \int_{0}^{s} e^{Y_{u}^{(t)}} d u$. For these family of Markov processes the general theory of stochastic processes can be applied to establish a recurrent integral equation of their Mellin transforms. Then, via Lemma 1, we can reverse the time once more and get the integral recurrent equation for Mellin transform of $I_{t}$.

In Theorem 1 we consider the case of $\alpha \geq 0$ and we give a recurrent integral equation for the Mellin transform of $I_{t}$. In the Corollaries 2 and 3 we consider the case when $X$ is a Levy process. We give the formulas for positive moments of $I_{t}$ and $I_{\infty}$ and also the formula for the Laplace transform. The results for $I_{\infty}$ coincide, of course, with the ones given in [7] for Levy subordinators. But it holds under less restrictive integrability conditions on $I_{\infty}$ and less restrictive condition on Levy measure at zero. We can also precise the number of finite moments of $I_{\infty}$ (cf. Corollary 3 and Corollary [5). In Theorem 2 and Corollaries 4 and 5 we present analogous result for the case $\alpha<0$.

\section{Finiteness of $\mathbf{E}\left(I_{t}^{\alpha}\right)$ FOR fixed $t>0$.}

In the following proposition we give simple sufficient conditions for the existence of the Mellin transform of $I_{t}$.

Proposition 1. Let $\alpha \in \mathbb{R}$ and $t>0$. The condition

$$
\int_{0}^{t} \int_{|x|>1} e^{-\alpha x} K_{s}(d x) d s<\infty,
$$

which is equivalent to

$$
\mathbf{E}\left(e^{-\alpha X_{t}}\right)<\infty
$$

implies that for $\alpha \geq 1$ and $\alpha \leq 0$

$$
\mathbf{E}\left(I_{t}^{\alpha}\right)<\infty .
$$

In addition, if (7) is valid for $\alpha=1$, then for $0 \leq \alpha \leq 1$ we have (9).

Remark 1. Assume that X has only positive jumps, then for $\alpha>0$ the condition (17) is always satisfied. In the same way, if $X$ has only negative jumps, then, of course, for $\alpha<0$ the condition (7) is satisfied. If $X$ has bounded jumps, the condition (7) is also satisfied. In general case, the condition (17) is equivalent to the one of the following conditions: if $\alpha>0$, then

$$
\int_{0}^{t} \int_{x<-1} e^{-\alpha x} K_{s}(d x) d s<\infty
$$


and if $\alpha<0$, then

$$
\int_{0}^{t} \int_{x>1} e^{-\alpha x} K_{s}(d x) d s<\infty
$$

In the case of Levy processes these conditions coincide with the ones given in [20], p. 79 .

Remark 2. It should be noticed that if the condition (7) is verified for $\alpha^{\prime}=\alpha+\delta$ with $\delta>0$ and $\alpha>0$, then it is verified also for $\alpha$. To see this, apply Hölder inequality to the integrals in (10) and (11) with the parameters $p=\frac{\alpha+\delta}{\alpha}$ and $q=\frac{\alpha+\delta}{\delta}$. In fact, since $\frac{1}{p}+\frac{1}{q}=1$ and $\nu([0, t] \times]-\infty,-1[)<\infty$ we have for (10):

$$
\begin{gathered}
\int_{0}^{t} \int_{x<-1} e^{-\alpha x} K_{s}(d x) d s \leq \\
\left(\int_{0}^{t} \int_{x<-1} e^{-\alpha p x} K_{s}(d x) d s\right)^{\frac{1}{p}}\left(\int_{0}^{t} \int_{x<-1} K_{s}(d x) d s\right)^{\frac{1}{q}}<\infty
\end{gathered}
$$

The same can be made for $\alpha<0$. In this case we take $\delta<0$.

Proof. We remark that for $t>0$, the law of $X_{t}$ coincide with the onedimensional law of Levy process $L$ with the triplet

$$
\left(\frac{1}{t} B_{t}, \frac{1}{t} C_{t}, \frac{1}{t} \int_{0}^{t} \int_{\mathbb{R} \backslash\{0\}} K_{s}(d x) d s\right)
$$

at the time $t$. Then, the equivalence of (17) and (8) is a simple consequence of Kruglov theorem (cf. Theorem 25.3 in [29]).

Let $\alpha \geq 1$ or $\alpha<0$ and $\tau$ be uniformly distributed on $[0, t]$ random variable independent of $X$ and. Then,

$$
\mathbf{E}\left(e^{-X_{\tau}}\right)=\mathbf{E}\left(I_{t} / t\right)
$$

and applying the Jensen inequality we get

$$
\left(I_{t} / t\right)^{\alpha} \leq \frac{1}{t} \int_{0}^{t} e^{-\alpha X_{s}} d s
$$

Now, due to (8) and (15), $f(s)=\mathbf{E}\left(e^{-\alpha X_{s}}\right), s \in[0, t]$, is well-defined continuous function, and, hence, by Fubini theorem

$$
\mathbf{E}\left(I_{t}^{\alpha}\right) \leq t^{\alpha-1} \int_{0}^{t} \mathbf{E}\left(e^{-\alpha X_{s}}\right) d s<\infty .
$$


If $\alpha=0$ the result is obvious, and if $0<\alpha<1$, then by the Hölder inequality we get that

$$
\mathbf{E}\left(I_{t}^{\alpha}\right) \leq\left(\mathbf{E} I_{t}\right)^{\alpha}<\infty
$$

and this ends the proof.

Let us give some examples useful in mathematical finance.

Example 1. Let $X$ be non-homogeneous Poisson process with intensity $\left(\lambda_{s}\right)_{s>0}$. Then, $K_{s}(A)=\lambda_{s} \cdot \delta_{\{1\}}(A)$ for all $A \in \mathcal{B}(\mathbb{R} \backslash\{0\})$, where $\delta_{\{1\}}$ is delta-function at 1 , and the condition (17) is satisfied as soon as $\int_{0}^{t} \lambda_{s} d s<\infty$. Hence, under the last condition, $\mathbf{E}\left(I_{t}^{\alpha}\right)<\infty$ for all $\alpha \in \mathbb{R}$.

Example 2. Let $L$ be Levy process with generating triplet $\left(b_{0}, c_{0}, K_{0}\right)$ and $X$ be the process $L$ time changed by deterministic continuously differentiable process $(\tau(t))_{t \geq 0}$, i.e. $X_{t}=L_{\tau(t)}$. Then, for $t \geq 0$

$$
\mathbf{E} e^{-\alpha L_{\tau(t)}}=e^{-\Phi(\alpha) \tau(t)}
$$

where $\Phi(\alpha)$ is Laplace exponent of $L$. We write

$\Phi(\alpha) \tau(t)=\alpha \tau(t) b_{0}-\frac{1}{2} \alpha^{2} \tau(t) c_{0}-\tau(t) \int_{\mathbb{R} \backslash\{0\}}\left(e^{-\alpha x}-1+\alpha x \mathbf{1}_{\{|x| \leq 1\}}\right) K_{0}(d x)$

and also, the Laplace exponent of $X$

$$
\Phi(t, \alpha)=\alpha B_{t}-\frac{1}{2} \alpha^{2} C_{t}-\int_{0}^{t} \int_{\mathbb{R} \backslash\{0\}}\left(e^{-\alpha x}-1+\alpha x \mathbf{1}_{\{|x| \leq 1\}}\right) K_{s}(d x) d s
$$

By the identification we get that that $B_{t}=b_{0} \tau(t), C_{t}=c_{0} \tau(t)$, and for all $A \in \mathcal{B}(\mathbb{R} \backslash\{0\}), \nu(A, d t)=K_{0}(A) \tau^{\prime}(t) d t$. Hence, $b_{t}=b_{0} \tau^{\prime}(t), c_{t}=$ $c_{0} \tau^{\prime}(t)$, and all $A \in \mathcal{B}(\mathbb{R} \backslash\{0\}), K_{t}(A)=K_{0}(A) \tau^{\prime}(t)$. The condition (7) is satisfied whenever

$$
\int_{|x|>1} e^{-\alpha x} K_{0}(d x)<\infty
$$

Under this condition $\mathbf{E}\left(I_{t}^{\alpha}\right)<\infty$.

Example 3. Let $L$ be Levy process with generating triplet $\left(b_{0}, c_{0}, K_{0}\right)$ such that $K_{0}$ has a density $f_{0}$ w.r.t. a Lebesgue measure and

$$
X_{t}=\int_{0}^{t} g_{s} d L_{s}
$$

where $g=\left(g_{s}\right)_{s \geq 0}$ is a not vanishing measurable square-integrable function. To find the characteristics of $X$ we take a canonical decomposition 
of $L$, namely

$$
L_{t}=b_{0} t+\sqrt{c_{0}} W_{t}+\int_{0}^{t} \int_{\mathbb{R} \backslash\{0\}} x\left(\mu_{X}(d s, d x)-\nu(d s, d x)\right)
$$

where $W$ is standard Brownian motion. We put this decomposition into the integral which defines $X$. We get by the identification that $B_{t}=b_{0} \int_{0}^{t} g_{s} d s, C_{t}=c_{0} \int_{0}^{t} g_{s}^{2} d s$. Moreover, since $\Delta X_{s}=g_{s} \Delta L_{s}$ for $s>0$, we deduce by projection theorem that for any positive measurable function $h$

$$
\mathbf{E} \int_{0}^{t} \int_{\mathbb{R} \backslash\{0\}} h(x) \mu_{X}(d s, d x)=\mathbf{E} \int_{0}^{t} \int_{\mathbb{R} \backslash\{0\}} h(x) K_{s}(d x) d s
$$

and that the l.h.s. of the previous equality is equal to

$$
\mathbf{E} \int_{0}^{t} \int_{\mathbb{R} \backslash\{0\}} h\left(x g_{s}\right) \mu_{L}(d s, d x)=\mathbf{E} \int_{0}^{t} \int_{\mathbb{R} \backslash\{0\}} h\left(x g_{s}\right) K_{0}(d x) d s
$$

Changing the variables we get that $K_{s}(d x)=\frac{1}{\left|g_{s}\right|} f_{0}\left(\frac{x}{g_{s}}\right) d x$ and that the condition (7) is satisfied whenever

$$
\int_{0}^{t} \int_{|x|>1} e^{-\alpha x g_{s}} K_{0}(d x) d s<\infty .
$$

Under the last condition, $\mathbf{E}\left(I_{t}^{\alpha}\right)<\infty$.

In what follows we assume that $K$ verify the following stronger relation then (6) : for $t>0$

$$
\int_{0}^{t} \int_{\mathbb{R} \backslash\{0\}}\left(x^{2} \wedge|x|\right) K_{s}(d x) d s<\infty .
$$

This supposition says, roughly speaking, that the "big" jumps of the process $X$ are integrable, and it ensures that finite variation part of semi-martingale decomposition of $X$ remains deterministic. Moreover, the truncation of the jumps is no more necessary. In addition, Kruglov theorem can be applied to show that (14) is equivalent to $\mathbf{E}\left(\left|X_{t}\right|\right)<\infty$ (cf. [29], Th. 25.3, p.159).

\section{Time REVERsal PROCEDURE}

We introduce, for fixed $t>0$, a new process $Y^{(t)}=\left(Y_{s}^{(t)}\right)_{0 \leq s \leq t}$ with $Y_{s}^{(t)}=X_{t}-X_{(t-s)-}$. To simplify the notations we anyway omit the index $(t)$ and write $Y_{s}$ instead of $Y_{s}^{(t)}$.

First of all we establish the relation between $I_{t}$ and the process $Y$. 
Lemma 1. For $t>0$ the following equality holds:

$$
I_{t}=e^{-Y_{t}} \int_{0}^{t} e^{Y_{s}} d s
$$

Proof. Using the definition of the process $Y$ and the assumption that $X_{0}=0$ we have

$$
\begin{aligned}
e^{-Y_{t}} \int_{0}^{t} e^{Y_{s}} d s & =\int_{0}^{t} e^{-Y_{t}+Y_{s}} d s=\int_{0}^{t} e^{-X_{(t-s)-}+X_{0}} d s \\
& =\int_{0}^{t} e^{-X_{t-s}} d s=\int_{0}^{t} e^{-X_{s}} d s=I_{t}
\end{aligned}
$$

since the integration of the both versions of the process w.r.t. the Lebesgue measure gives the same result.

We will show that the process $Y$ is PII and we will give its semimartingale triplet with respect to its natural filtration. For that we put

$$
\bar{b}_{u}= \begin{cases}b_{t-u} & \text { for } 0 \leq u<t \\ b_{t} & \text { for } u=t\end{cases}
$$

which can be written also as

$$
\bar{b}_{u}=\mathbf{1}_{\{t\}}(u)\left(b_{t}-b_{0}\right)+b_{t-u},
$$

where $\mathbf{1}_{\{t\}}$ is indicator function of the set $\{t\}$. We do the similar definitions for $\bar{c}$ and $\bar{K}$ :

$$
\begin{gathered}
\bar{c}_{u}=\mathbf{1}_{\{t\}}(u)\left(c_{t}-c_{0}\right)+c_{t-u}, \\
\bar{K}_{u}(A)=\mathbf{1}_{\{t\}}(u)\left(K_{t}(A)-K_{0}(A)\right)+K_{t-u}(A)
\end{gathered}
$$

for all $A \in \mathcal{B}(\mathbb{R} \backslash\{0\})$.

Lemma 2. The process $Y$ is a process with independent increments, it is a semi-martingale with respect to its natural filtration, and its semi-martingale triplet $(\bar{B}, \bar{C}, \bar{\nu})$ is given by :

$$
\bar{B}_{s}=\int_{0}^{s} \bar{b}_{u} d u, \bar{C}_{s}=\int_{0}^{s} \bar{c}_{u} d u, \bar{\nu}(d s, d x)=\bar{K}_{s}(d x) d s,
$$

where $0 \leq s \leq t$.

Proof Let us take $0=s_{0}<s_{1}<s_{2}<\cdots<s_{n}=t$ with $n \geq 2$. Then the increments $\left(Y_{s_{k}}-Y_{s_{k-1}}\right)_{1 \leq k \leq n}$ of the process $Y$ are equal to $\left(X_{\left(t-s_{k-1}\right)-}-X_{\left(t-s_{k}\right)-}\right)_{1 \leq k \leq n}$ and $0=t-s_{n}<t-s_{n-1}<\cdots<t-s_{0}=t$. From the fact that $X$ is the process with independent increments, the characteristic function of the vector $\left(X_{t-s_{k-1}-h}-X_{t-s_{k}-h}\right)_{1 \leq i \leq n}$ with 
small $h>0$, can be written as a product of the corresponding characteristic functions. Namely, for any real constants $\left(\lambda_{k}\right)_{0 \leq k \leq n}$ we get:

$\mathbf{E} \exp \left(i \sum_{k=1}^{n} \lambda_{k}\left(X_{t-s_{k-1}-h}-X_{t-s_{k}-h}\right)\right)=\prod_{k=1}^{n} \mathbf{E} \exp \left(i \lambda_{k}\left(X_{t-s_{k-1}-h}-X_{t-s_{k}-h}\right)\right)$

Then, passing to the limit as $h \rightarrow 0+$,

$\mathbf{E} \exp \left(i \sum_{k=1}^{n} \lambda_{k}\left(X_{\left(t-s_{k-1}\right)-}-X_{\left(t-s_{k}\right)-}\right)\right)=\prod_{k=1}^{n} \mathbf{E} \exp \left(i \lambda_{k}\left(X_{\left(t-s_{k-1}\right)-}-X_{\left(t-s_{k}\right)-}\right)\right)$

Hence, $Y$ is a process with independent increments.

We know that we can identify the semi-martingale characteristics w.r.t. the natural filtration of the process with independent increments from the characteristic function of this process. We notice that $Y_{s}=X_{t}-$ $X_{(t-s)-}$ and by the independence of $Y_{s}$ and $X_{(t-s)-}$

$$
\mathbf{E}\left(e^{i \lambda X_{t}}\right)=\mathbf{E}\left(e^{i \lambda Y_{s}}\right) \mathbf{E}\left(e^{i \lambda X_{(t-s)-}}\right)
$$

Then,

$$
\begin{gathered}
\mathbf{E} \exp \left(i \lambda Y_{s}\right)=\mathbf{E} \exp \left(i \lambda X_{t}\right) / \mathbf{E} \exp \left(i \lambda X_{(t-s)-}\right)= \\
\exp \left\{i \lambda \int_{t-s}^{t} b_{u} d u-\frac{1}{2} \lambda^{2} \int_{t-s}^{t} c_{u} d u+\int_{t-s}^{t} \int_{\mathbb{R} \backslash\{0\}}\left(e^{i \lambda x}-1-i \lambda x\right) K_{u}(d x) d u\right\}
\end{gathered}
$$

We substitute $u$ by $u^{\prime}=t-u$ in the integrals to obtain

$$
\begin{gathered}
\operatorname{E} \exp \left(i \lambda Y_{s}\right)= \\
\exp \left\{i \lambda \int_{0}^{s} \bar{b}_{u} d u-\frac{1}{2} \lambda^{2} \int_{0}^{s} \bar{c}_{u} d u+\int_{0}^{s} \int_{\mathbb{R} \backslash\{0\}}\left(e^{i \lambda x}-1-i \lambda x\right) \bar{K}_{u}(d x) d u\right\}
\end{gathered}
$$

Therefore, the characteristics of $Y$ are as in (15) and the proof is complete. $\square$

\section{ReCURRENT Formulas For the Mellin transform of $I_{t}$ WITH $\alpha \geq 0$.}

Let us consider two important processes related with the process $Y$, namely the process $V=\left(V_{s}\right)_{0 \leq s \leq t}$ and $J=\left(J_{s}\right)_{0 \leq s \leq t}$ defined via

$$
V_{s}=e^{-Y_{s}} J_{s}, \quad J_{s}=\int_{0}^{s} e^{Y_{u}} d u
$$

We underline that the both processes depend of the parameter $t$. 
We remark that according to Lemma 1 , $I_{t}=V_{t}$ for each $t \geq 0$. For $\alpha \geq 0$ and $t \geq 0$ we introduce the Mellin transform of $I_{t}$ of the parameter $\alpha$ :

$$
m_{t}^{(\alpha)}=\mathbf{E}\left(I_{t}^{\alpha}\right)=\mathbf{E}\left(e^{-\alpha Y_{t}} J_{t}^{\alpha}\right)
$$

and the Mellin transform for shifted process:

$$
m_{s, t}^{(\alpha)}=\mathbf{E}\left[\left(\int_{s}^{t} e^{-\left(X_{u}-X_{s-}\right)} d u\right)^{\alpha}\right]
$$

Notice that $m_{0, t}^{(\alpha)}=m_{t}^{(\alpha)}$. Notice also that

$$
m_{s, t}^{(\alpha)}=\mathbf{E}\left[\left(\int_{s}^{t} e^{-\left(X_{u}-X_{s}\right)} d u\right)^{\alpha}\right]
$$

In fact, $X_{u}-X_{s}=X_{u}-X_{s-}-\Delta X_{s}$ and

$$
\int_{s}^{t} e^{-\left(X_{u}-X_{s-}\right)} d u=e^{-\Delta X_{s}} \int_{s}^{t} e^{-\left(X_{u}-X_{s}\right)} d u
$$

Since $\Delta X_{s}$ and $\left(X_{u}-X_{s}\right)_{u \geq s}$ are independent, and $\mathbf{E}\left(e^{-\alpha \Delta X_{s}}\right)=1$, we get the equality of two expressions for $m_{s, t}^{(\alpha)}$.

We introduce also two functions: for $0 \leq s \leq t$

$$
H_{s}^{(\alpha)}=\alpha b_{s}-\frac{1}{2} \alpha^{2} c_{s}-\int_{\mathbb{R} \backslash\{0\}}\left(e^{-\alpha x}-1+\alpha x\right) K_{s}(d x)
$$

and

$$
\bar{H}_{s}^{(\alpha)}=\mathbf{1}_{\{t\}}(s)\left(H_{t}^{(\alpha)}-H_{0}^{(\alpha)}\right)+H_{t-s}^{(\alpha)}
$$

These functions represent the derivatives w.r.t. $s$, of the Laplace exponents $\Phi(s, \alpha)$ and $\bar{\Phi}(s, \alpha)$. We recall that

$$
\Phi(s, \alpha)=\alpha B_{s}-\frac{\alpha^{2}}{2} C_{s}-\int_{0}^{s} \int_{\mathbb{R} \backslash\{0\}}\left(e^{-\alpha x}-1+\alpha x\right) \nu(d u, d x)
$$

and

$$
\bar{\Phi}(s, \alpha)=\alpha \bar{B}_{s}-\frac{\alpha^{2}}{2} \bar{C}_{s}-\int_{0}^{s} \int_{\mathbb{R} \backslash\{0\}}\left(e^{-\alpha x}-1+\alpha x\right) \bar{\nu}(d u, d x)
$$

where $\nu$ and $\bar{\nu}$ are the compensators of the jump measure of $X$ and $Y$ respectively. We notice, that these functions are well-defined under condition (7). We also notice that

$$
\bar{\Phi}(s, \alpha)=\Phi(t, \alpha)-\Phi(t-s, \alpha)
$$

Our aim now is to obtain a recurrent integral equation for the Mellin transform of $I_{t}$. For condition (18) below see Remarks 1 and 2 , 
Theorem 1. Let $\alpha \geq 1$ be fixed and assume that $t>0$. Suppose that (5) and (14) hold and there exists $\delta>0$ such that

$$
\int_{0}^{t} \int_{x<-1} e^{-(\alpha+\delta) x} K_{s}(d x) d s<\infty .
$$

Then, $m_{t}^{(\alpha)}$ is well-defined and the following recurrent integral equation holds

$$
m_{t}^{(\alpha)}=\alpha \int_{0}^{t} m_{u, t}^{(\alpha-1)} e^{-\Phi(u, \alpha)} d u
$$

If $X$ is Levy process, then for all $t>0$

$$
m_{t}^{(\alpha)}=\alpha e^{-\Phi(\alpha) t} \int_{0}^{t} m_{s}^{(\alpha-1)} e^{\Phi(\alpha) s} d s
$$

Moreover,

$$
\frac{d}{d t}\left[m_{t}^{(\alpha)}\right]=-m_{t}^{(\alpha)} \Phi(\alpha)+\alpha m_{t}^{(\alpha-1)}
$$

Proof. From Lemma 2 we know that $Y=\left(Y_{s}\right)_{0 \leq s \leq t}$ is a process with independent increments which is a semi-martingale, i.e. $Y_{s}=\bar{B}_{s}+$ $\bar{M}_{s}$, where $\bar{B}$ is a deterministic process of finite variation on finite intervals and $\bar{M}$ is a local martingale. But the local martingales with independent increments are always the martingales (see [31]).

For $n \geq 1$ we introduce the stopping times

$$
\tau_{n}=\inf \left\{0 \leq s \leq t: V_{s} \geq n \text { or } \exp \left(-Y_{s}\right) \geq n\right\}
$$

with $\inf \{\emptyset\}=+\infty$. For fixed $s, 0<s<t$, we write the Ito formula for $V_{s \wedge \tau_{n}}^{\alpha}$ :

$$
\begin{array}{r}
V_{s \wedge \tau_{n}}^{\alpha}=\alpha \int_{0}^{s \wedge \tau_{n}} V_{u-}^{\alpha-1} d V_{u}+\frac{1}{2} \alpha(\alpha-1) \int_{0}^{s \wedge \tau_{n}} V_{u-}^{\alpha-2} d<V^{c}>_{u} \\
\quad+\int_{0}^{s \wedge \tau_{n}} \int_{\mathbb{R} \backslash\{0\}}\left(\left(V_{u-}+x\right)^{\alpha}-V_{u-}^{\alpha}-\alpha V_{u-}^{\alpha-1} x\right) \mu_{V}(d u, d x)
\end{array}
$$

where $\mu_{V}$ is the measure of the jumps of $V$. Using integration by part formula, we have:

$$
d V_{u}=d u+J_{u} d\left(e^{-Y_{u}}\right)
$$

Now, again by the Ito formula, we get

$$
e^{-Y_{u}}=e^{-Y_{0}}-\int_{0}^{u} e^{-Y_{v-}} d Y_{v}+\frac{1}{2} \int_{0}^{u} e^{-Y_{v-}} d<Y^{c}>_{v}
$$




$$
+\int_{0}^{u} \int_{\mathbb{R} \backslash\{0\}} e^{-Y_{v-}}\left(e^{-x}-1+x\right) \mu_{Y}(d v, d x)
$$

Then, putting (24) into (23), we obtain

$$
\begin{gathered}
d V_{u}^{c}=-e^{-Y_{u-}} J_{u} d Y_{u}^{c}=-V_{u-} d Y_{u}^{c}, \\
d<V^{c}>_{u}=V_{u-}^{2} d<Y^{c}>_{u}
\end{gathered}
$$

and

$$
\Delta V_{u}=e^{-Y_{u-}} J_{u}\left(e^{-\Delta Y_{u}}-1\right)=V_{u-}\left(e^{-\Delta Y_{u}}-1\right),
$$

where $\Delta V_{u}=V_{u}-V_{u-}$ and $\Delta Y_{u}=Y_{u}-Y_{u-}$. The previous relations imply that

$$
\begin{gathered}
V_{s \wedge \tau_{n}}^{\alpha}=\alpha \int_{0}^{s \wedge \tau_{n}} V_{u-}^{\alpha-1} d u \\
+\alpha \int_{0}^{s \wedge \tau_{n}} J_{u} V_{u-}^{\alpha-1} d\left(e^{-Y_{u}}\right)+\frac{1}{2} \alpha(\alpha-1) \int_{0}^{s \wedge \tau_{n}} V_{u-}^{\alpha} d<Y^{c}>_{u} \\
+\int_{0}^{s \wedge \tau_{n}} \int_{\mathbb{R} \backslash\{0\}} V_{u-}^{\alpha}\left(e^{-\alpha x}-1-\alpha\left(e^{-x}-1\right)\right) \mu_{Y}(d u, d x)
\end{gathered}
$$

Now, to use in efficient way the Ito formula for $e^{-Y_{u}}$ given before, we introduce the processes $A=\left(A_{u}\right)_{0 \leq u \leq t}$ and $N=\left(N_{u}\right)_{0 \leq u \leq t}$ via

$$
\begin{aligned}
& A_{u}=\int_{0}^{u} e^{-Y_{v-}}\left[-d \bar{B}_{v}+\frac{1}{2} d \bar{C}_{v}\right]+\int_{0}^{u} \int_{\mathbb{R} \backslash\{0\}} e^{-Y_{v-}}\left(e^{-x}-1+x\right) \bar{\nu}(d v, d x) \\
& N_{u}=-\int_{0}^{u} e^{-Y_{v}-} d \bar{M}_{v}+\int_{0}^{u} \int_{\mathbb{R} \backslash\{0\}} e^{-Y_{v-}}\left(e^{-x}-1+x\right)\left[\mu_{Y}(d v, d x)-\bar{\nu}(d v, d x)\right]
\end{aligned}
$$

We notice that $A$ is a process of locally bounded variation and $N$ is a local martingale with localizing sequence $\left(\tau_{n}\right)_{n \geq 1}$, since $\bar{B}, \bar{C}$ are of bounded variation on bounded intervals and

$$
\int_{0}^{u} \int_{\mathbb{R} \backslash\{0\}}\left(e^{-x}-1+x\right) \bar{K}_{s}(d x) d s<\infty .
$$

From (24) we get that

$$
e^{-Y_{u}}=e^{-Y_{0}}+A_{u}+N_{u} .
$$

We incorporate this semi-martingale decomposition into (27) and we consider its martingale part. This martingale part is represented by the term

$$
\begin{gathered}
\alpha \int_{0}^{s \wedge \tau_{n}} V_{u-}^{\alpha-1} J_{u} d N_{u}= \\
\alpha \int_{0}^{s \wedge \tau_{n}} V_{u-}^{\alpha}\left[-d \bar{M}_{u}+\int_{\mathbb{R} \backslash\{0\}}\left(e^{-x}-1+x\right)\left(\mu_{Y}(d v, d x)-\bar{\nu}(d v, d x)\right)\right]
\end{gathered}
$$


which is a local martingale. Let $\left(\tau_{n}^{\prime}\right)_{n \geq 0}$ be a localizing sequence for this local martingale and let $\bar{\tau}_{n}=\tau_{n} \wedge \tau_{n}^{\prime}$. Then we do additional stopping with $\tau_{n}^{\prime}$ in previous expressions, and we take mathematical expectation. Using the fact that the expectations of martingales starting from zero are equal to zero and also applying the projection theorem, we obtain:

$$
\begin{gathered}
\mathbf{E}\left(V_{s \wedge \bar{\tau}_{n}}^{\alpha}\right)=\alpha \mathbf{E}\left(\int_{0}^{s \wedge \bar{\tau}_{n}} V_{u-}^{\alpha-1} d u\right) \\
+\alpha \mathbf{E}\left(\int_{0}^{s \wedge \bar{\tau}_{n}} V_{u-}^{\alpha-1} J_{u} d A_{u}\right)+\frac{1}{2} \alpha(\alpha-1) \mathbf{E}\left(\int_{0}^{s \wedge \bar{\tau}_{n}} V_{u-}^{\alpha} d \bar{C}_{u}\right) \\
+\mathbf{E}\left(\int_{0}^{s \wedge \bar{\tau}_{n}} \int_{\mathbb{R} \backslash\{0\}} V_{u-}^{\alpha}\left[e^{-\alpha x}-1-\alpha\left(e^{-x}-1\right)\right] \bar{\nu}(d u, d x)\right)
\end{gathered}
$$

and, hence,

$$
\mathbf{E}\left(V_{s \wedge \bar{\tau}_{n}}^{\alpha}\right)=\alpha \mathbf{E}\left(\int_{0}^{s \wedge \bar{\tau}_{n}} V_{u}^{\alpha-1} d u\right)-\mathbf{E}\left(\int_{0}^{s \wedge \tau_{n}} V_{u-}^{\alpha} d \bar{\Phi}(u, \alpha)\right)
$$

We remark that $\bar{\tau}_{n} \rightarrow+\infty(\mathbf{P}-$ a.s. $)$ as $n \rightarrow+\infty$. To pass to the limit as $n \rightarrow \infty$ in r.h.s. of the above equality, we use the Lebesgue monotone convergence theorem for the first term and the Lebesgue dominated convergence theorem for the second term. In fact, for the second term we have using (16) and (17) :

$$
\left|\int_{0}^{s \wedge \bar{\tau}_{n}} V_{u-}^{\alpha} d \bar{\Phi}(u, \alpha)\right| \leq \int_{0}^{t} V_{u-}^{\alpha}\left|\bar{H}_{u}^{(\alpha)}\right| d u
$$

In addition,

$$
\mathbf{E}\left(\int_{0}^{t} V_{u-}^{\alpha}\left|\bar{H}_{u}^{(\alpha)}\right| d u\right) \leq \sup _{0 \leq u \leq t} \mathbf{E}\left(V_{u}^{\alpha}\right) \int_{0}^{t}\left|\bar{H}_{u}^{(\alpha)}\right| d u
$$

The function $\left(\bar{H}_{u}^{(\alpha)}\right)_{0 \leq u \leq t}$ is deterministic function, integrable on finite intervals. Hence, it remains to show that

$$
\sup _{0 \leq s \leq t} \mathbf{E}\left(V_{s}^{\alpha}\right)<\infty
$$

By the Jensen inequality similar to (12) we obtain :

$$
V_{s}^{\alpha} \leq s^{\alpha-1} \int_{0}^{s} e^{\alpha\left(Y_{u}-Y_{s}\right)} d u
$$

and due to the fact that $Y_{u}-Y_{s}=X_{(t-s)-}-X_{(t-u)-}$, we get

$$
\mathbf{E}\left(V_{s}^{\alpha}\right) \leq s^{\alpha-1} \int_{0}^{s} \mathbf{E}\left(e^{\alpha\left(X_{t-s}-X_{t-u}\right)}\right) d u
$$


Since the process $X$ is a process with independent increments, we have for $0 \leq u \leq s \leq t$

$$
\begin{gathered}
\mathbf{E}\left(e^{\alpha\left(X_{t-s}-X_{t-u}\right)}\right)=\mathbf{E}\left(e^{-\alpha X_{t-u}}\right) / \mathbf{E}\left(e^{-\alpha X_{t-s}}\right)= \\
\exp \left\{-\int_{0}^{t-u} H_{r}^{(\alpha)} d r+\int_{0}^{t-s} H_{r}^{(\alpha)} d r\right\} \leq \exp \left\{\int_{0}^{t}\left|H_{r}^{(\alpha)}\right| d r\right\}
\end{gathered}
$$

Due to the Remark1 and the condition (18), $H^{(\alpha)}$ is integrable function on finite intervals, and hence,

$$
\sup _{0 \leq s \leq t} \mathbf{E}\left(V_{s}^{\alpha}\right) \leq t^{\alpha} \exp \left\{\int_{0}^{t}\left|H_{r}^{(\alpha)}\right| d r\right\}<\infty
$$

To pass to the limit in the 1.h.s. of (30), we show that the family of $\left(V_{s \wedge \tau_{n}}^{\alpha}\right)_{n \geq 1}$ is uniformly integrable, uniformly in $0 \leq s \leq t$. For that we recall that $Y_{s}=\bar{B}_{s}+\bar{M}_{s}$ where $\bar{B}$ is a drift part and $\bar{M}$ is a martingale part of $Y$, and we introduce

$$
\bar{V}_{s}=e^{-\bar{M}_{s}} \int_{0}^{s} e^{\bar{M}_{u}} d u
$$

Then,

$$
\begin{gathered}
V_{s}=e^{-Y_{s}} \int_{0}^{s} e^{Y_{u}} d u=e^{-\bar{M}_{s}-\bar{B}_{s}} \int_{0}^{s} e^{\bar{M}_{u}+\bar{B}_{u}} d u \\
\leq \sup _{0 \leq u \leq s}\left(e^{\bar{B}_{u}-\bar{B}_{s}}\right) \bar{V}_{s} \leq e^{\operatorname{Var}(\bar{B})_{t}} \bar{V}_{s}
\end{gathered}
$$

where $\operatorname{Var}(\bar{B})_{t}$ is the variation of $\bar{B}$ on the interval $[0, t]$. This quantity is deterministic and bounded, hence, it is sufficient to prove uniform integrability of $\left(\bar{V}_{s \wedge \tau_{n}}^{\alpha}\right)_{n \geq 1}$.

Next, we show that $\left(\bar{V}_{s}\right)_{0 \leq s \leq t}$ is a submartingale w.r.t. a natural filtration of $Y$. Let $s^{\prime}>s$, then

$$
\begin{gathered}
\mathbf{E}\left(\bar{V}_{s^{\prime}} \mid \mathcal{F}_{s}\right)=\mathbf{E}\left(e^{-\bar{M}_{s^{\prime}}} \int_{0}^{s^{\prime}} e^{\bar{M}_{u}} d u \mid \mathcal{F}_{s}\right)= \\
\mathbf{E}\left(e^{-\left(\bar{M}_{s^{\prime}}-\bar{M}_{s}\right)}\left[\bar{V}_{s}+e^{-\bar{M}_{s}} \int_{s}^{s^{\prime}} e^{\bar{M}_{u}} d u\right] \mid \mathcal{F}_{s}\right) \geq \\
\mathbf{E}\left(e^{-\left(\bar{M}_{s^{\prime}}-\bar{M}_{s}\right)} \bar{V}_{s} \mid \mathcal{F}_{s}\right)=\bar{V}_{s} \mathbf{E}\left(e^{-\left(\bar{M}_{s^{\prime}}-\bar{M}_{s}\right)}\right)
\end{gathered}
$$

The expression for $\mathbf{E}\left(e^{-\left(\bar{M}_{s^{\prime}}-\bar{M}_{s}\right)}\right)$ can be find from the expression of the characteristic exponent of $Y$ without its drift part:

$$
\begin{aligned}
& \mathbf{E}\left(e^{-\left(\bar{M}_{s^{\prime}}-\bar{M}_{s}\right)}=\right. \\
& \quad \exp \left(\frac{1}{2} \int_{s}^{s^{\prime}} c_{u} d u+\int_{s}^{s^{\prime}} \int_{\mathbb{R} \backslash\{0\}}\left(e^{-x}-1+x\right) \bar{\nu}(d u, d x)\right) \geq 1 .
\end{aligned}
$$


Then, $\left(\bar{V}_{s}^{\alpha}\right)_{0 \leq s \leq t}$ is a submartingale, and by Doob stopping theorem $(P$-a.s. $)$

$$
\mathbf{E}\left(\bar{V}_{s}^{\alpha} \mid \mathcal{F}_{s \wedge \tau_{n}}\right) \geq \bar{V}_{s \wedge \tau_{n}}^{\alpha} .
$$

Hence, for all $n \geq 1, c>0$ and $\mathbf{I}(\cdot)$ indicator function

$$
\begin{aligned}
& \mathbf{E}\left(\bar{V}_{s \wedge \tau_{n}}^{\alpha} \mathbf{I}_{\left\{\bar{V}_{s \wedge \tau_{n}}>c\right\}}\right) \leq \mathbf{E}\left(\mathbf{E}\left(\bar{V}_{s}^{\alpha} \mid \mathcal{F}_{s \wedge \tau_{n}}\right) \mathbf{I}_{\left\{\mathbf{E}\left(\bar{V}_{s}^{\alpha} \mid \mathcal{F}_{s \wedge \tau_{n}}\right)>c\right\}}\right) \leq \\
& c^{-\frac{\delta}{\alpha}} \mathbf{E}\left(\mathbf{E}\left(\bar{V}_{s}^{\alpha} \mid \mathcal{F}_{s \wedge \tau_{n}}\right)^{\frac{\alpha+\delta}{\alpha}}\right) \leq c^{-\frac{\delta}{\alpha}} \mathbf{E}\left(\bar{V}_{s}^{\alpha+\delta}\right)
\end{aligned}
$$

Finally, we show in the same way as the proof of (31) that

$$
\sup _{0 \leq s \leq t} \mathbf{E}\left(\bar{V}_{s}^{\alpha+\delta}\right)<\infty
$$

and it proves the uniform integrability of the family $\left(V_{s \wedge \tau_{n}}^{\alpha}\right)_{n \geq 1}$ uniformly in $0 \leq s \leq t$.

After limit passage, we get that

$$
\mathbf{E}\left(V_{s}^{\alpha}\right)=-\int_{0}^{s} \mathbf{E}\left(V_{u}^{\alpha}\right) d \bar{\Phi}(u, \alpha)+\alpha \int_{0}^{s} \mathbf{E}\left(V_{u}^{\alpha-1}\right) d u
$$

We see that each term of this equation is differentiable w.r.t. $s$ for $s<t$. In fact, the family $\left(V_{s}^{\alpha}\right)_{0 \leq s \leq t}$ is uniformly integrable and the function $\left(\mathbf{E}\left(V_{s}^{\alpha}\right)\right)_{0 \leq s \leq t}$ is continuous in $s$ as well as $(\bar{\Phi}(s, \alpha))_{0 \leq s<t}$. We calculate the derivatives in $s$ of both sides of the above equation and we solve the corresponding linear equation to obtain:

$$
\mathbf{E}\left(V_{s}^{\alpha}\right)=\alpha e^{-\bar{\Phi}(s, \alpha)} \int_{0}^{s} \mathbf{E}\left(V_{u}^{\alpha-1}\right) e^{\bar{\Phi}(u, \alpha)} d u
$$

Now, we write that $\bar{\Phi}(u, \alpha)-\bar{\Phi}(s, \alpha)=-\Phi(t-u, \alpha)+\Phi(t-s, \alpha)$ and we let $s \rightarrow t-$ to get

$$
\mathbf{E}\left(V_{t}^{\alpha}\right)=\alpha \int_{0}^{t} \mathbf{E}\left(V_{u}^{\alpha-1}\right) e^{-\Phi(t-u, \alpha)} d u
$$

Notice that $I_{t}=V_{t}$ and, hence, $\mathbf{E}\left(V_{t}^{\alpha}\right)=m_{t}^{(\alpha)}$. Since

$$
V_{u}=\int_{0}^{u} e^{Y_{v}-Y_{u}} d v=\int_{0}^{u} e^{-\left(X_{(t-v)-}-X_{(t-u)-}\right)} d v=\int_{t-u}^{t} e^{-\left(X_{v}-X_{(t-u)-}\right)} d v
$$

we also have $\mathbf{E}\left(V_{u}^{\alpha-1}\right)=m_{t-u, t}^{(\alpha-1)}$. Then, we obtain (19) after the change of variables in integrals replacing $t-u$ by $u$.

In particular case of Levy process, we use (19) and we take into account that $m_{u, t}^{(\alpha-1)}=m_{t-u}^{(\alpha-1)}$ and that $\Phi(u, \alpha)=\Phi(\alpha) u$. Then, after the change of variables, the formula (20) follows as well as (21).

Remark 3. It should be noticed that the relation (19) can be obtained with another technique (see [27]), based on the approach of [7]. 
Now we will apply our results to calculate the moments.

Example 4. We consider Levy process $L=\left(L_{t}\right)_{t \geq 0}$ with generating triplet $\left(b_{0}, c_{0}, K_{0}\right)$ starting from 0 , and time changed by deterministic process $\left(\tau_{t}\right)_{t \geq 0}$ with $\tau_{t}=r \ln (1+t)$ with $r>0$, i.e. $X_{t}=L_{\tau_{t}}$ for $t \geq 0$. Then, by change of variables $u=r \ln (1+s)$ we get

$$
I_{t}=\int_{0}^{t} e^{-L_{r \ln (1+s)}} d s=\frac{1}{r} \int_{0}^{r \ln (1+t)} e^{-\left(L_{u}-u / r\right)} d u=\frac{1}{r} \int_{0}^{r \ln (1+t)} e^{-\tilde{L}_{u}} d u
$$

where $\tilde{L}$ is Levy process with generating triplet $\left(b_{0}-\frac{1}{r}, c_{0}, K_{0}\right)$. We denote by $\tilde{\Phi}$ the Laplace exponent of $\tilde{L}$. Then for $k \geq 0, \tilde{\Phi}(k)=$ $\Phi(k)-\frac{k}{r}$ and

$$
\mathbf{E}\left(I_{t}\right)=\frac{1}{r} \int_{0}^{r \ln (1+t)} e^{-\tilde{\Phi}(1) u} d u= \begin{cases}\frac{1-(1+t)-r \Phi(1)+1}{r \Phi(1)-1} & \text { if } r \Phi(1)-1 \neq 0 \\ \ln (1+t) & \text { otherwise. }\end{cases}
$$

For shifted process $X_{u}^{(s)}=L_{\tau_{u}}-L_{\tau_{s}}, u \geq s$ the corresponding moments

$$
m_{s, t}^{(n)}=\mathbf{E}\left[\left(\int_{s}^{t} e^{-\left(L_{\tau_{u}}-L_{\tau_{s}}\right)} d u\right)^{n}\right]=\mathbf{E}\left[\left(\int_{s}^{t} e^{-L_{\left(\tau_{u}-\tau_{s}\right)}} d u\right)^{n}\right]
$$

since $L$ is homogeneous process and $\left(L_{\tau_{u}}-L_{\tau_{s}}\right)_{u \geq s} \underline{\mathcal{L}}\left(L_{\tau_{u}-\tau_{s}}\right)_{u \geq s}$.

We change the variable putting $\tau_{u}-\tau_{s}=r \ln (1+u)-r \ln (1+s)=v-s$ where $v$ is new variable. We denote $v(s, t)=r \ln \left(\frac{1+t}{1+s}\right)$ and we remark that $1+u=(1+s) \exp ((v-s) / r)$. Then,

$$
\begin{gathered}
\mathbf{E}\left[\left(\int_{s}^{t} e^{-\left(L_{\tau_{u}-\tau_{s}}\right)} d u\right)^{n}\right]=\mathbf{E}\left[\left(\frac{1+s}{r} \int_{s}^{s+v(s, t)} e^{-\tilde{L}_{v-s}} d v\right)^{n}\right] \\
=\frac{(1+s)^{n}}{r^{n}} \mathbf{E}\left[\left(\int_{0}^{v(s, t)} e^{-\tilde{L}_{u}} d u\right)^{n}\right]
\end{gathered}
$$

Finally, we get for $n \geq 0$ :

$$
m_{s, t}^{(n)}=\frac{(1+s)^{n}}{r^{n}} \tilde{m}_{v(s, t)}^{(n)}
$$

where $\tilde{m}_{v(s, t)}^{(n)}$ is n-th moment of the exponential functional of $\tilde{L}$ on $[0, v(s, t)]$. We suppose for simplicity that $\tilde{\Phi}$ is strictly monotone on the interval $[0, n+1]$. Then, using the integral equation of Theorem 1 and the expression for the moments of Levy processes given in Corollary 1 below, and the fact that

$$
e^{-\Phi(s, n+1)}=e^{-\Phi(n+1) \tau_{s}}=(1+s)^{-r \Phi(n+1)}
$$


we get for $n \geq 1$ :

$$
m_{t}^{(n+1)}=\frac{(n+1) !}{r^{n}} \sum_{k=0}^{n-1} \int_{0}^{t}(1+s)^{q(n)} \frac{e^{-\tilde{\Phi}(k) v(s, t)}-e^{-\tilde{\Phi}(n) v(s, t)}}{\prod_{0 \leq i \leq n, i \neq k}(\tilde{\Phi}(i)-\tilde{\Phi}(k))} d s
$$

where $q(n)=n-r \Phi(n+1)$ and $\tilde{\Phi}(k)$ is the Laplace exponent of Levy process $\tilde{L}, \tilde{\Phi}(k)=\Phi(k)-\frac{k}{r}, 1 \leq k \leq n$. To express the final result we put

$$
\rho(k)=r \Phi(k)-k, \quad \gamma(n, k)=n-k-r(\Phi(n+1)-\Phi(k))
$$

and

$$
Q_{t}(n, k)=\frac{(1+t)^{\gamma(n, k)+1}-1}{(\gamma(n, k)+1)(1+t)^{\rho(k)}}
$$

Then, after the integration we find that for $n \geq 1$

$$
\mathbf{E}\left(I_{t}^{n+1}\right)=m_{t}^{(n+1)}=(n+1) ! \sum_{k=0}^{n-1} \frac{Q_{t}(n, k)-Q_{t}(n, n)}{\prod_{0 \leq i \leq n, i \neq k}(\rho(i)-\rho(k))}
$$

Remark 4. It is clear that the explicit formulas for the moments in non-homogeneous case will be rather exceptional. For numerical calculus the following formula could be useful: for all $0 \leq s \leq t$

$$
m_{s, t}^{(\alpha)}=\alpha \int_{s}^{t} m_{u, t}^{(\alpha-1)} e^{\Phi(u, \alpha)-\Phi(s, \alpha)} d u
$$

To obtain this formula it is sufficient to find the Laplace exponent of shifted process $\left(X_{u}^{(s)}\right)_{s \leq u \leq t}$ with $X_{u}^{(s)}=X_{u}-X_{s}$. Since $X$ is a PII, the variables $X_{s}$ and $X_{u}-X_{s}$ are independent, and

$$
\mathbf{E}\left(e^{-\alpha X_{u}}\right)=\mathbf{E}\left(e^{-\alpha X_{s}}\right) \mathbf{E}\left(e^{-\alpha\left(X_{u}-X_{s}\right)}\right)
$$

and then, the Laplace exponent of shifted process $X^{(s)}$ is given by:

$$
\Phi^{(s)}(u, \alpha)=\Phi(u, \alpha)-\Phi(s, \alpha) .
$$

\section{Positive moments of $I_{t}$ And $I_{\infty}$ FOR Levy PRocesses}

Now we suppose that $X$ is Levy process with the parameters $\left(b_{0}, c_{0}, K_{0}\right)$. In this case the relation (14) become:

$$
\int_{\mathbb{R} \backslash\{0\}}\left(x^{2} \wedge|x|\right) K_{0}(d x)<\infty .
$$


It should be noticed that in [7] the condition on Levy measure was

$$
\int_{\mathbb{R} \backslash\{0\}}(|x| \wedge 1) K_{0}(d x)<\infty
$$

and this condition is stronger at zero and weaker at infinity then (34). When $X$ is Levy process, the condition (18) become: there exists $\delta>0$

$$
\int_{x<-1} e^{-(\alpha+\delta) x} K_{0}(d x)<\infty .
$$

Corollary 1. Let $n \geq 1$ and suppose that $\Phi$ is bijective on $[0, n] \cap \mathbb{N}$. Then,

$$
\mathbf{E}\left(I_{t}^{n}\right)=n ! \sum_{k=0}^{n-1} \frac{e^{-\Phi(k) t}-e^{-\Phi(n) t}}{\prod_{0 \leq i \leq n, i \neq k}(\Phi(i)-\Phi(k))}
$$

Proof. The formula follows from (20) by induction.

We present here two examples, one of them is related with Brownian motion, and second one with compound Poisson process.

Example 5. Let $X$ be a Brownian motion with drift $\mu>0$ and diffusion coefficient $\sigma>0$, i.e. $X_{t}=\mu t+\sigma W_{t}$ where $W=\left(W_{t}\right)_{t \geq 0}$ is standard Brownian motion. Then, $\Phi(\alpha)=\alpha \mu-\frac{\alpha^{2} \sigma^{2}}{2}$ and if $\frac{2 \mu}{\sigma^{2}}$ is not an integer, we get:

$$
\mathbf{E}\left(I_{t}^{n}\right)=n ! \sum_{k=0}^{n-1} \frac{e^{-\left(k \mu-k^{2} \sigma^{2} / 2\right) t}-e^{-\left(n \mu-n^{2} \sigma^{2} / 2\right) t}}{\prod_{0 \leq i \leq n, i \neq k}(i-k)\left(\mu-(i+k) \sigma^{2} / 2\right)}
$$

Example 6. Let $X$ be compound Poisson process such that $X_{t}=$ $\sum_{k=1}^{N_{t}} U_{k}$ where $\left(U_{k}\right)_{k \geq 0}$ is a sequence of independent random variables with distribution function $F$ and $N$ is a homogeneous Poisson process with intensity $\lambda>0$. Then, $\Phi(\alpha)=\lambda \int_{\mathbb{R} \backslash\{0\}}\left(1-e^{-\alpha x}\right) F(d x)$. In particular, if the $U_{k}$ 's are standard normal variables, we get that $\Phi(\alpha)=$ $\lambda\left(1-e^{\alpha^{2} / 2}\right)$ and

$$
\mathbf{E}\left(I_{t}^{n}\right)=n ! \sum_{k=0}^{n-1} \frac{\exp \left(-\lambda t\left(1-e^{k^{2} / 2}\right)\right)-\exp \left(-\lambda t\left(1-e^{n^{2} / 2}\right)\right)}{\lambda^{n} \prod_{0 \leq i \leq n, i \neq k}\left(e^{k^{2} / 2}-e^{i^{2} / 2}\right)}
$$


We introduce the Laplace-Carson transform $\hat{m}_{q}^{(\alpha)}$ of $m_{t}^{(\alpha)}$ of the parameter $q>0$ :

$$
\hat{m}_{q}^{(\alpha)}=\int_{0}^{\infty} q e^{-q t} m_{t}^{(\alpha)} d t
$$

This integral is always well-defined in general sense, since the integrand is positive.

Corollary 2. (cf. [7]) Let $X$ be a Levy process which verifies (34) and (36), and such that for fixed $\alpha \geq 1, m_{\infty}^{(\alpha)}<\infty$. Then the LaplaceCarson transforms $\hat{m}_{q}^{(\alpha)}$ and $\hat{m}_{q}^{(\alpha-1)}$ of $m_{t}^{(\alpha)}$ and $m_{t}^{(\alpha-1)}$ respectively, are well-defined and we have a recurrent formula:

$$
\hat{m}_{q}^{(\alpha)}(q+\Phi(\alpha))=\alpha \hat{m}_{q}^{(\alpha-1)}
$$

In particular, for integer $n \geq 1$ such that $m_{\infty}^{(n)}<\infty$ we get:

$$
\hat{m}_{q}^{(n)}=\frac{n !}{\prod_{k=1}^{n}(q+\Phi(k))}
$$

As a consequence,

$$
\mathbf{E}\left(I_{\infty}^{n}\right)=\frac{n !}{\prod_{k=1}^{n} \Phi(k)}
$$

Moreover, if all positive moments of $I_{\infty}$ exist and the series below converges, then the Laplace transform of $I_{\infty}$ of parameter $\beta \geq 0$ is given by:

$$
\mathbf{E}\left(e^{-\beta I_{\infty}}\right)=\sum_{n=0}^{\infty} \frac{(-1)^{n} \beta^{n}}{\prod_{k=1}^{n} \Phi(k)}
$$

Proof. The first equality for the Laplace-Carson transforms follows directly from (21). The second equality can be obtained as particular case from the first one, by recurrence.

For the third one we prove that $\hat{m}_{q}^{(n)} \rightarrow m_{\infty}^{(n)}$ as $q \rightarrow 0$. In fact, $m_{t}^{(n)} \rightarrow m_{\infty}^{(n)}$ as $t \rightarrow+\infty$ and

$$
\lim _{t \rightarrow+\infty} \frac{1}{t} \int_{0}^{t} m_{s}^{(n)} d s=m_{\infty}^{(n)} .
$$

Let us denote $M_{t}^{(n)}=\int_{0}^{t} m_{s}^{(n)} d s$. By integration by part formula we have for each $t>0$ :

$$
\int_{0}^{t} q e^{-q s} m_{s}^{(n)} d s=\left[q e^{-q t} M_{t}^{(n)}\right]_{0}^{t}+\int_{0}^{t} q^{2} e^{-q s} M_{s}^{(n)} d s
$$


Then, since $M_{0}^{(n)}=0$ and $M_{t}^{(n)} / t \rightarrow m_{\infty}^{(n)}$ as $t \rightarrow+\infty$,

$$
\hat{m}_{q}^{(n)}=\int_{0}^{\infty} q e^{-q s} m_{s}^{(n)} d s=q^{2} \int_{0}^{\infty} e^{-q s} M_{s}^{(n)} d s
$$

Since $q^{2} \int_{0}^{\infty} s e^{-q s} d s=1$, we get

$$
\hat{m}_{q}^{(n)}-m_{\infty}^{(n)}=q^{2} \int_{0}^{\infty} e^{-q s}\left(M_{s}^{(n)}-s m_{\infty}^{(n)}\right) d s
$$

For each $\epsilon>0$ there exists $t_{\epsilon}$ such that for $s \geq t_{\epsilon},\left|\frac{M_{s}^{(n)}}{s}-m_{\infty}^{(n)}\right| \leq \epsilon$. Then,

$$
\begin{aligned}
& \left|\hat{m}_{q}^{(n)}-m_{\infty}^{(n)}\right| \leq q^{2} \int_{0}^{t_{\epsilon}} e^{-q s}\left|M_{s}^{(n)}-s m_{\infty}^{(n)}\right| d s+ \\
& \quad q^{2} \int_{t_{\epsilon}}^{\infty} s e^{-q s}\left|\frac{M_{s}^{(n)}}{s}-m_{\infty}^{(n)}\right| d s \leq q^{2} \int_{0}^{t_{\epsilon}} e^{-q s}\left|M_{s}^{(n)}-s m_{\infty}^{(n)}\right| d s+\epsilon
\end{aligned}
$$

We notice that

$$
\lim _{q \rightarrow 0} q^{2} \int_{0}^{t_{\epsilon}} e^{-q s}\left|M_{s}^{(n)}-s m_{\infty}^{(n)}\right| d s=0
$$

Then, taking $\lim _{\epsilon \rightarrow 0} \lim _{q \rightarrow 0}$ in the previous inequality we get that

$$
\hat{m}_{q}^{(n)} \rightarrow m_{\infty}^{(n)}
$$

as $q \rightarrow 0$. Finally, we take the limit as $q \rightarrow 0$ in second equality, to obtain the third one. The formula for the Laplace transform of $I_{\infty}$ can be proved by using Taylor expansion with remainder in Lagrange form.

Example 7. Let $X$ be homogeneous Poisson process of intensity $\lambda>0$. Then all positive moments of $I_{\infty}$ exist, and we have for $0 \leq \beta<\lambda$

$$
\mathbf{E}\left(e^{-\beta I_{\infty}}\right)=\sum_{n=0}^{\infty} \frac{(-1)^{n} \beta^{n}}{\lambda^{n} \prod_{k=1}^{n}\left(1-e^{-k}\right)}
$$

Corollary 3. Let $\alpha_{0}=\inf \{\alpha>0 \mid \Phi(\alpha) \leq 0\}$ with $\inf \{\emptyset\}=+\infty$. Then, $\mathbf{E}\left(I_{\infty}^{n}\right)<\infty$ if and only if $1 \leq n<\alpha_{0}$. In particular, for Brownian motion with drift coefficient $b_{0}$ and diffusion coefficient $c_{0} \neq 0$,

$$
\Phi(n)=n b_{0}-\frac{1}{2} n^{2} c_{0}
$$

and the moment of $I_{\infty}$ of order $n \geq 1$ will exist if $n<\frac{2 b_{0}}{c_{0}}$.

If $X$ is a subordinator with non-zero Levy measure $K_{0}$ such that (35) holds, then

$$
\Phi(n)=n\left[b_{0}-\int_{\mathbb{R}^{+} \backslash\{0\}} x K_{0}(d x)\right]-\int_{\mathbb{R}^{+} \backslash\{0\}}\left(e^{-n x}-1\right) K_{0}(d x),
$$


and under the condition

$$
b_{0}-\int_{\mathbb{R}^{+} \backslash\{0\}} x K_{0}(d x) \geq 0,
$$

all moments of $I_{\infty}$ exist.

Proof. Let $n=\sup \left\{k \geq 1: \mathbf{E}\left(I_{\infty}^{k}\right)<\infty\right\}$. If $n=+\infty$, then $\Phi(k)>0$ for all $k \geq 1$ and $\alpha_{0}=+\infty$. If $1 \leq n<+\infty$, from Corollary 2 we get that $\Phi(n)>0$. Since $\Phi(\alpha)$ is concave function such that $\Phi(0)=0$, we conclude that $n<\alpha_{0}$. Conversely, substituting $t-s$ by $s$ in (20) of Theorem 1 we get:

$$
\mathbf{E}\left(I_{t}^{n}\right) \leq n \mathbf{E}\left(I_{\infty}^{n-1}\right) \int_{0}^{t} e^{-\Phi(n) s} d s
$$

If $1 \leq n<\alpha_{0}, \Phi(n)>0$, and the integral on the r.h.s. of this inequality converge as $t \rightarrow \infty$. By induction, it gives that $\mathbf{E}\left(I_{\infty}^{n}\right)<\infty$. Moreover, the results for continuous case and the case when $X$ is a subordinator, follow immediately from the expression of $\Phi(k)$.

Example 8. Let $X$ be time changed Brownian motion, namely $X_{t}=$ $\mu \tau_{t}+\sigma W_{\tau_{t}}$ where $W=\left(W_{t}\right)_{t \geq 0}$ is standard Brownian motion, $\mu \in \mathbb{R}$, $\sigma>0$ and $\tau_{t}$ is first hitting time of the level $t$ of the independent (from $W$ ) standard Brownian motion $B=\left(B_{t}\right)_{t \geq 0}$ with the drift coefficient $b>0$. Then, as known, $\Phi(\alpha)=\left(b^{2}+2 \alpha \mu-\alpha^{2} \sigma^{2}\right)^{1 / 2}-b$ with $b^{2}+2 \alpha \mu-$ $\alpha^{2} \sigma^{2}>0$ (see for instance [8], formula 2.0.1, p.295). Then, $\mathbf{E}\left(I_{\infty}^{n}\right)<\infty$ if and only if $2 \mu-n \sigma>0$.

Example 9. Let $X$ be pure discontinuous Levy process with Levy measure

$$
K_{0}(d x)=\frac{c \exp (-M x)}{x^{1+\beta}} \mathbf{I}_{0,+\infty[}(x) d x
$$

where $c>0, M>0,0<\beta<1$. Then,

$$
\Phi(\alpha)=\frac{c \Gamma(1-\beta)}{-\beta}\left((M+\alpha)^{\beta}-M^{\beta}-\alpha M^{\beta-1} \beta\right) .
$$

Then, $\Phi(\alpha)>0$ for $\alpha \geq 1$, and all moments of $I_{\infty}$ exist.

\section{Recurrent formulas for the Mellin transform of $I_{t}$ WITH $\alpha<0$.}

In the following Theorem 2, we derive the integro-differential equation for the Mellin transform $m_{t}^{(\alpha)}$ of $I_{t}$ with $\alpha<0$. 
Theorem 2. Let $\alpha<0$ and $t>0$ be fixed. Suppose that (5) and(14) hold and

$$
\int_{0}^{t} \int_{x>1} e^{(|\alpha|+1) x} K_{s}(d x) d s<\infty .
$$

Then, for $s>0, m_{s, t}^{(\alpha)}$ is well-defined as well as $m_{s, t}^{(\alpha-1)}$ and we get the following recurrent differential equation:

$$
m_{s, t}^{(\alpha-1)}=\frac{1}{\alpha}\left(m_{s, t}^{(\alpha)} H_{s}^{(\alpha)}-\frac{d}{d s} m_{s, t}^{(\alpha)}\right)
$$

In the case of Levy process $X$ we have:

$$
m_{s}^{(\alpha-1)}=\frac{1}{\alpha}\left(m_{s}^{(\alpha)} \Phi(\alpha)+\frac{d}{d s} m_{s}^{(\alpha)}\right)
$$

Proof. The proof of this result is similar to the proof of Theorem 1 , For $n \geq 1$ we introduce the stopping times

$$
\tau_{n}=\inf \left\{u \geq s: V_{u} \leq \frac{1}{n} \text { or } \exp \left(-Y_{u}\right) \geq n\right\}
$$

with $\inf \{\emptyset\}=+\infty$. Then from the Ito formula similarly to (44) we get: for $0<s<t$

$$
\begin{aligned}
V_{t \wedge \tau_{n}}^{\alpha}= & V_{s}^{\alpha}+\alpha \int_{s}^{t \wedge \tau_{n}} V_{u-}^{\alpha-1} d V_{u}+\frac{1}{2} \alpha(\alpha-1) \int_{s}^{t \wedge \tau_{n}} V_{u-}^{\alpha-2} d<V^{c}>_{u} \\
& +\int_{s}^{t \wedge \tau_{n}} \int_{\mathbb{R} \backslash\{0\}}\left(\left(V_{u-}+x\right)^{\alpha}-V_{u-}^{\alpha}-\alpha V_{u-}^{\alpha-1} x\right) \mu_{V}(d u, d x)
\end{aligned}
$$

where $\mu_{V}$ is the measure of the jumps of $V$. Using (23), (25), (26) we have

$$
\begin{gathered}
V_{t \wedge \tau_{n}}^{\alpha}=V_{s}^{\alpha}+\alpha \int_{s}^{t \wedge \tau_{n}} V_{u-}^{\alpha-1} d u \\
+\alpha \int_{s}^{t \wedge \tau_{n}} J_{u} V_{u-}^{\alpha-1} d\left(e^{-Y_{u}}\right)+\frac{1}{2} \alpha(\alpha-1) \int_{s}^{t \wedge \tau_{n}} V_{u-}^{\alpha} d<Y^{c}>_{u} \\
+\int_{s}^{t \wedge \tau_{n}} \int_{\mathbb{R} \backslash\{0\}} V_{u-}^{\alpha}\left(e^{-\alpha x}-1-\alpha\left(e^{-x}-1\right)\right) \mu_{Y}(d u, d x)
\end{gathered}
$$

where $\mu_{Y}$ the measure of the jumps of $Y$. Taking in account (28) we, finally, find that

$$
\mathbf{E}\left(V_{t \wedge \tau_{n}}^{\alpha}\right)=\mathbf{E}\left(V_{s}^{\alpha}\right)+\alpha \mathbf{E}\left(\int_{s}^{t \wedge \tau_{n}} V_{u-}^{\alpha-1} d u\right)-\mathbf{E}\left(\int_{s}^{t \wedge \tau_{n}} V_{u-}^{\alpha} d \bar{\Phi}(u, \alpha)\right)
$$


We remark that $\tau_{n} \rightarrow+\infty(\mathbf{P}-$ a.s. $)$ as $n \rightarrow+\infty$. To pass to the limit as $n \rightarrow \infty$ in the r.h.s. of the above equality, we use the Lebesgue monotone convergence theorem for the first term and the Lebesgue dominated convergence theorem for the second term. In fact, for second term we have:

$$
\left|\int_{s}^{t \wedge \tau_{n}} V_{u-}^{\alpha} d \bar{\Phi}(u, \alpha)\right| \leq \int_{s}^{t} V_{u-}^{\alpha}\left|\bar{H}_{u}^{(\alpha)}\right| d u
$$

In addition,

$$
\mathbf{E}\left(\int_{s}^{t} V_{u-}^{\alpha}\left|\bar{H}_{u}^{(\alpha)}\right| d u\right) \leq \sup _{s \leq u \leq t} \mathbf{E}\left(V_{u}^{\alpha}\right) \int_{s}^{t}\left|\bar{H}_{u}^{(\alpha)}\right| d u
$$

The function $\left(\bar{H}_{u}^{(\alpha)}\right)_{0 \leq u \leq t}$ is deterministic function, integrable on finite intervals. Hence, it remains to show that

$$
\sup _{s \leq u \leq t} \mathbf{E}\left(V_{u}^{\alpha}\right)<\infty
$$

By the Jensen inequality

$$
V_{u}^{\alpha} \leq u^{\alpha-1} \int_{0}^{u} e^{\alpha\left(Y_{v}-Y_{u}\right)} d v
$$

Then, in the same way as in Theorem 1 we get that

$$
\sup _{s \leq u \leq t} \mathbf{E}\left(V_{u}^{\alpha}\right) \leq s^{\alpha} \exp \left\{\int_{0}^{t}\left|H_{r}^{(\alpha)}\right| d r\right\}
$$

To pass to the limit in the 1.h.s. of (45), we prove that the family of $\left(V_{u \wedge \tau_{n}}^{\alpha}\right)_{n \geq 1}$ is uniformly integrable, uniformly in $u \in[s, t]$ in the same way as in Theorem 1. For that we introduce a submartingale $\left(\bar{V}_{u}^{(\alpha)}\right)_{s \leq u \leq t}$ with

$$
\bar{V}_{u}^{(\alpha)}=e^{-\alpha \bar{M}_{u}} \int_{0}^{u} e^{\alpha \bar{M}_{v}} d v
$$

We remark that

$$
V_{u}^{\alpha} \leq e^{|\alpha| \operatorname{Var}(\bar{B})_{t}} \bar{V}_{u}^{(\alpha)} .
$$

In addition, on the set $\left\{\bar{V}_{u \wedge \tau_{n}}^{(\alpha)}>c\right\}$ we have: $1<\left(\frac{\bar{V}_{u \wedge \tau_{n}}^{(\alpha)}}{c}\right)^{\frac{1}{|\alpha|}}$. Then

$$
\bar{V}_{u \wedge \tau_{n}}^{(\alpha)} \mathbf{I}_{\left\{\bar{V}_{u \wedge \tau_{n}}^{(\alpha)}>c\right\}} \leq\left(\bar{V}_{u \wedge \tau_{n}}^{(\alpha)}\right)^{1+\frac{1}{|\alpha|}} c^{-\frac{1}{|\alpha|}}=\left(\bar{V}_{u \wedge \tau_{n}}^{(\alpha)}\right)^{\frac{\alpha-1}{\alpha}} c^{-\frac{1}{|\alpha|}}
$$

Applying Jensen inequality we get that

$$
\left(\bar{V}_{u \wedge \tau_{n}}^{(\alpha)}\right)^{\frac{\alpha-1}{\alpha}} \leq \bar{V}_{u \wedge \tau_{n}}^{(\alpha-1)} u^{\frac{1}{|\alpha|}}
$$

Hence, we proved that for all $n \geq 1, c>0$ and $s \leq u \leq t$

$$
\mathbf{E}\left(\bar{V}_{u \wedge \tau_{n}}^{(\alpha)} \mathbf{I}_{\left\{\bar{V}_{u \wedge \tau_{n}}^{(\alpha)}>c\right\}}\right) \leq c^{-\frac{1}{|\alpha|}} \max \left(s^{\frac{1}{|\alpha|}}, t^{\frac{1}{|\alpha|}}\right) \mathbf{E}\left(\bar{V}_{u}^{(\alpha-1)}\right)
$$


Finally, we prove that

$$
\sup _{s \leq u \leq t} \mathbf{E}\left(\bar{V}_{u}^{(\alpha-1)}\right)<\infty
$$

in the same manner as before, and it implies the uniform integrability.

After limit passage we get that

$$
\mathbf{E}\left(V_{t}^{\alpha}\right)=\mathbf{E}\left(V_{s}^{\alpha}\right)-\int_{s}^{t} \mathbf{E}\left(V_{u}^{\alpha}\right) d \bar{\Phi}(u, \alpha)+\alpha \int_{s}^{t} \mathbf{E}\left(V_{u}^{\alpha-1}\right) d u
$$

We differentiate each term of this equality w.r.t. $s$ to obtain that

$$
\frac{d}{d s} \mathbf{E}\left(V_{s}^{\alpha}\right)+\mathbf{E}\left(V_{s}^{\alpha}\right) \bar{H}_{s}^{(\alpha)}-\alpha \mathbf{E}\left(V_{s}^{\alpha-1}\right)=0
$$

We take in account that $\mathbf{E}\left(V_{s}^{\alpha}\right)=m_{t-s, t}^{(\alpha)}, \mathbf{E}\left(V_{s}^{\alpha-1}\right)=m_{t-s, t}^{(\alpha-1)}$ and that $\bar{H}_{s}^{(\alpha)}=H_{t-s}^{(\alpha)}$. This implies that

$$
\frac{d}{d s} m_{t-s, t}^{(\alpha)}+m_{t-s, t}^{(\alpha)} H_{t-s}^{(\alpha)}-\alpha m_{t-s, t}^{(\alpha-1)}=0
$$

Finally, replacing $t-s$ by $s$ we get (41). In the case of Levy processes $m_{t-s, t}^{(\alpha)}=m_{s}^{(\alpha)}$ due to homogeneity, and $H_{t-s}^{(\alpha)}=\Phi(\alpha)$, and this gives (42).

To present the results about negative moments of Levy process $X$ with the parameters $\left(b_{0}, c_{0}, K_{0}\right)$, we introduce the condition:

$$
\int_{x>1} e^{(|\alpha|+1) x} K_{0}(d x)<\infty .
$$

Corollary 4. (cf. [7])Let $X$ be Levy process verifying (34) and (48), and let $\alpha \leq-1$ be fixed. Suppose that $m_{\infty}^{(\alpha-1)}<\infty$. Then the LaplaceCarson transforms of $m_{\infty}^{(\alpha)}$ and $m_{\infty}^{(\alpha-1)}$ are well-defined and we have a recurrent formula:

$$
\hat{m}_{q}^{(\alpha-1)}=\frac{1}{\alpha}\left(\hat{m}_{q}^{(\alpha)} \Phi(\alpha)+q \hat{m}_{q}^{(\alpha)}\right)
$$

and, hence,

$$
\hat{m}_{q}^{(\alpha-1)}=\frac{1}{\alpha} \hat{m}_{q}^{(\alpha)}(q+\Phi(\alpha))
$$

In particular, under above conditions, for integer $n \geq 2$ and $\alpha=-n$ we get:

$$
\hat{m}_{q}^{(-n)}=\hat{m}_{q}^{(-1)} \cdot \frac{(-1)^{n-1}}{(n-1) !} \prod_{k=1}^{n-1}(q+\Phi(-k))
$$


As a consequence,

$$
\mathbf{E}\left(I_{\infty}^{-n}\right)=\mathbf{E}\left(I_{\infty}^{-1}\right) \cdot \frac{(-1)^{n-1}}{(n-1) !} \prod_{k=1}^{n-1} \Phi(-k)
$$

Proof. Two first equalities given above follow directly from Theorem 2. The third one can be proved in the same way as in Corollary 2 by letting $q \rightarrow 0$.

Example 10. For compound Poisson process presented in Example 6 with $U_{k}$ 's which follows standard normal distribution, we get for $n \geq 1$

$$
\mathbf{E}\left(I_{\infty}^{-n}\right)=\mathbf{E}\left(I_{\infty}^{-1}\right) \frac{\lambda^{n-1}}{(n-1) !} \prod_{k=1}^{n-1}\left(e^{\frac{k^{2}}{2}}-1\right)
$$

Corollary 5. Let $\beta=\sup \{k \geq 1 \mid-\infty<\Phi(-l)<0$ for $1 \leq l \leq k\}$ with $\sup \{\emptyset\}=1$. Then $\mathbf{E}\left(I_{\infty}^{-(n+1)}\right)<\infty$ if and only if $n \leq \beta$ and $\mathbf{E}\left(I_{\infty}^{-1}\right)<\infty$.

In particular, for Brownian motion with the drift coefficient $b_{0}$ and the diffusion coefficient $c_{0} \neq 0, \mathbf{E}\left(I_{\infty}^{-(n+1)}\right)<\infty$ if and only if $\frac{2 b_{0}}{c_{0}}>-1$.

If $X$ is a subordinator with $\int_{\mathbb{R}^{+} \backslash\{0\}} x K_{0}(d x)<\infty$, then

$$
\Phi(-k)=-k\left[b_{0}-\int_{\mathbb{R}^{+} \backslash\{0\}} x K_{0}(d x)\right]-\int_{\mathbb{R}^{+} \backslash\{0\}}\left(e^{k x}-1\right) K_{0}(d x),
$$

and under the condition

$$
b_{0}-\int_{\mathbb{R}^{+} \backslash\{0\}} x K_{0}(d x) \geq 0
$$

$\mathbf{E}\left(I_{\infty}^{-(n+1)}\right)<\infty$ if and only if $\int_{\mathbb{R}^{+} \backslash\{0\}}\left(e^{n x}-1\right) K_{0}(d x)<\infty$.

Proof. Suppose that $\mathbf{E}\left(I_{\infty}^{-(n+1)}\right)<\infty$ for some $n>0$. Then by CauchySchwartz inequality we get that for all $k, 1 \leq k \leq n, \mathbf{E}\left(I_{\infty}^{-k}\right)<\infty$. Then the formula (51) yields that $\mathbf{E}\left(I_{\infty}^{-1}\right)<\infty$ and $-\infty<\Phi(-k)<0$ for $1 \leq k \leq n$. Hence, $n \leq \beta$ and $\mathbf{E}\left(I_{\infty}^{-1}\right)<\infty$.

Conversely, if $n \leq \beta$ and $\mathbf{E}\left(I_{\infty}^{-1}\right)<\infty$, then $-\infty<\Phi(-k)<0$ for $1 \leq k \leq n$. Then from (42) we deduce that

$$
m_{s}^{-(k+1)}=\frac{\Phi(-k)}{-k} m_{s}^{(-k)}-\frac{1}{k} \frac{d}{d s} m_{s}^{(-k)} \leq \frac{\Phi(-k)}{-k} m_{s}^{(-k)} \leq \frac{|\Phi(-k)|}{k} m_{\infty}^{(k)}
$$

since $\frac{d}{d s} m_{s}^{(-k)} \geq 0$. Hence,

$$
\mathbf{E}\left(I_{\infty}^{-(n+1)}\right)=m_{\infty}^{-(n+1)} \leq \prod_{k=1}^{n} \frac{|\Phi(-k)|}{k} \mathbf{E}\left(I_{\infty}^{-1}\right)<\infty .
$$


In the case of Brownian motion we conclude that $\Phi(-k)=-k b_{0}-$ $\frac{1}{2} k^{2} c_{0}>-\infty$ for all $k \geq 1$. Since $\Phi$ is concave function with $\Phi(0)=0$, the condition $\Phi(-1)<0$ ensures the existence of all negative moments. In the case when $X$ is a subordinator, and under mentioned condition (52), all $\Phi(-k)<0$ and only the condition of finiteness of $\Phi(-k)$ is involved in the existence of the negative moments.

Example 11. Let us apply the Corollary 5 to time changed Brownian motion considered in Example 8. We get that $\Phi(\alpha)<0$ whenever $-b^{2} \leq 2 \alpha \mu-\alpha^{2} \sigma^{2}<0$. Hence, all negative moments of $I_{\infty}$ exists if $\mathbf{E}\left(I_{\infty}^{-1}\right)<\infty$ and $2 \mu+\sigma^{2}>0$.

\section{Acknowledgement}

We are grateful to our referees for very useful remarks and comments.

\section{REFERENCES}

[1] S. Asmussen. Ruin probabilities, World Scientific, 2000.

[2] O. Barndorff-Nielsen, A.N. Shiryaev. Change of Time and Change of Measure. World Scientific, 2010.

[3] A. Behme (2015) Exponential functionals of Lévy Processes with Jumps, ALEA, Lat. Am. J. Probab. Math. Stat. 12 (1), 375-397.

[4] A. Behme, A. Lindner (2015) On exponential functionals of Levy processes, Journal of Theoretical Probability 28, 681-720.

[5] J. Bertoin. Lévy processes, Cambridge University Press, 1996, p.266.

[6] J. Bertoin, A. Lindler, R. Maller (2008) On continuity Properties of the Law of Integrals of Levy Processes, In Séminaire de probabilités XLI, 1934, 137-159.

[7] J. Bertoin, M. Yor (2005) Exponential functionals of Levy processes, Probability Surveys, 191-212.

[8] A. Borodin, P. Salminen. Handbook of Brownian motion - Facts and Formulae, Birkhäuser Verlag, Basel-Boston-Berlin, 2002, 672p.

[9] P. Carmona, F. Petit, M. Yor (1997) On the distribution and asymptotic results for exponential functionals of Levy processes, In "Exponential functionals and principal values related to Brownian motion", 73-130. Biblioteca de la Revista Matematica IberoAmericana.

[10] P. Carr, L. Wu. (2004) Time-changed Lévy processes and option pricing, Journal of Financial Economics 71, 113-141.

[11] D. Dufresne (1990) The distribution of a perpetuity, with applications to risk theory and pension funding. Scand. Actuarial J., 1-2, 39-79.

[12] I. Epifani, A. Lijoi, I Prünster (2003) Exponential functionals and mean of neutral-to-the-right priors, Biometrika, 90, 4, 791-808.

[13] K.B. Erickson, R. Maller (2004) Generalised Ornstein-Uhlenbeck processes and the convergence of Lévy integrals., p. 70-94. In : Séminaire de probabilités, Lect. Notes Math. 1857, Springer, Berlin. 
[14] H.K. Gjessing, J. Paulsen (1997) Present value distributions with applications to ruin theory and stochastic equations, Stochastic Process. Appl. 71 (1), 123144.

[15] J. Jacod, A. Shiryaev. Limit theorems for Stochastic Processes, SpringerVerlag, 1987, 606p.

[16] M. Jeanblanc, M. Yor, M. Chesnay. Mathematical Methods for Financial Markets, Springer Finance Textbook, 2009, 332p.

[17] Yu. Kabanov, S. Pergamentshchikov (2016) In the insurance business risky investment are dangerous: the case of negative risk sums, Finance and Stochastics, 20, 2, 355-379.

[18] C. Kardaras, S. Robertson (2014) Continuous time perpetuities and time reversal of diffusions ARXIV: 1411.7551v1.

[19] A. Kuznetsov, J.C. Prado, M.Savov (2012) Distributional properties of exponential functionals of Levy processes, Electron. J. Probab. 8, 1-35.

[20] A. Kyprianou (2014) Fluctuations of Lévy processes with applications, SpringerVerlag, Berlin, Heildelberg, second addition, 2014.

[21] J. Lamperti (1972) Semi-stable Markov Processes. Z. Wahrscheinlichkeitstheorie verv. Gebiete, 22, 205-225.

[22] J. C. Pardo, V. Rivero, K. Van Schaik (2013) On the density of exponential functionals of Lévy processes, Bernoulli, 1938-1964.

[23] A. A. Novikov, N. E. Kordzahiya (2012 ) Pitman estimators: an asymptotic variance revisited, Teor. Veroyatnost. i Primenen., Volume 57, Issue 3, 603-611.

[24] P. Patie, M. Savov(2016) Bernstein-Gamma functions and exponential functionals of Lévy processes, arXiv:1604.05960v2.

[25] J. Paulsen (2008) Ruin models with investment income, Probability Surveys, vol. 5, 416-434.

[26] P. Salminen, O. Wallin. (2005) Perpetual integral functional of diffusions and their numerical computations, Dept. of Math, Univ. of Oslo, Pure Mathematics, 35.

[27] P. Salminen, L. Vostrikova (2016) On moments of exponential functionals of additive processes, Peprint.

[28] P. Salminen, M. Yor. (2005) Perpetual Integral Functionals as Hitting and Occupation Times, Electronic Journal of Probability, Vol. 10, Issue 11, 371-419.

[29] K. Sato. Lévy Processes and Infinitely Divisible Distributions, Cambridge University Press, second edition, 2013.

[30] A. N. Shiryaev. Essentials of Stochastic Finance: Facts, Models, Theory, World Scientific, 1999, p. 834.

[31] A. N. Shiryaev, A. S. Cherny (2002) Vector Stochastic Integrals and the Fundamental Theorems of Asset Pricing, Proc. Steklov Inst. Math. 237, 6-49. 